\title{
Development of a Novel MR Colonography via Iron-Based Solid Lipid Nanoparticles
}

\author{
Huiyang Wang',*, Siqi Wang ${ }^{2, *}$, Xisong Zhu ${ }^{3}$, Wenxiu Ding ${ }^{4}$, Tianlun Shen ${ }^{2}$, Hongjie Fan', \\ Yanhua Zhang ${ }^{5}$, Lijun Peng ${ }^{6}$, Hong Yuan $\mathbb{D}^{6}$, Xiangrui Liư ${ }^{7}$, Jun Ling ${ }^{2}$, Jihong Sun'
}

'Department of Radiology, Sir Run Run Shaw Hospital, School of Medicine, Zhejiang University, Hangzhou, 3 I00I6, People's Republic of China; ${ }^{2}$ MOE Key Laboratory of Macromolecular Synthesis and Functionalization, Department of Polymer Science and Engineering, Zhejiang University, Hangzhou, 310027, People's Republic of China; ${ }^{3}$ Department of Radiology, Quzhou Central Hospital Affiliated to Zhejiang Chinese Medical University, Quzhou, 324002, People's Republic of China; ${ }^{4}$ Department of Ultrasound Medicine, The First Affiliated Hospital, School of Medicine, Zhejiang University, Hangzhou, 310003, People's Republic of China; ${ }^{5}$ Department of Pathology, Sir Run Run Shaw Hospital, School of Medicine, Zhejiang University, Hangzhou, 310016, People's Republic of China; ${ }^{6}$ College of Pharmaceutical Sciences, Zhejiang University, Hangzhou, 310030, People's Republic of China; ${ }^{7}$ Department of Pharmacology, Zhejiang University School of Medicine, Hangzhou, 310058, People's Republic of China

*These authors contributed equally to this work

Correspondence: Jihong Sun, Department of Radiology, Sir Run Run Shaw Hospital, School of Medicine, Zhejiang University, Hangzhou, 3I00I6, People's Republic of China, Tel + 13857176538, Fax +57I-86006762, Email sunjihong@zju.edu.cn; Jun Ling, MOE Key Laboratory of Macromolecular Synthesis and Functionalization, Department of Polymer Science and Engineering, Zhejiang University, Hangzhou, 3 I0027, People's Republic of China, Tel + |36457|730I, Fax +57I-87953739, Email lingjun@zju.edu.cn

Purpose: To develop an iron-based solid lipid nanoparticle (SLN) absorbable by the intestinal wall and assess the differential diagnostic value of intestinal lesions in magnetic resonance imaging (MRI).

Methods: SLNs were prepared with the simultaneous loading of trivalent $\mathrm{Fe}$ ions $\left(\mathrm{Fe}^{3+}\right)$, levodopa methyl ester (DM), and fluorescein isothiocyanate (FITC). We evaluated the particle size, loading rate, encapsulation efficiency, and cytotoxicity of SLNs. The $\mathrm{T}_{1}$ contrast effects of the FeDM-FITC-SLNs and gadolinium-based contrast agent (GBCA) were compared in different mouse models: acute ulcerative colitis (AUC), chronic ulcerative colitis (CUC), colon adenocarcinoma (COAD), and normal control. MRI was performed in the same mouse with intravenous injection of GBCA on day 1 and enema of FeDM-FITC-SLNs on day 2. The signal-to-noise ratios (SNRs) were compared using one-way analysis of variance. Tissues were then collected for histology.

Results: The average particle size of FeDM-FITC-SLN was $220 \mathrm{~nm}$. The mean FeDM loading rate was 94.3\%, and the encapsulation efficiency was $60.3 \%$. The relaxivity was $4.02 \mathrm{mM}^{-1} \cdot \mathrm{s}^{-1}$. After enema with FeDM-FITC-SLNs, MRI showed the following contrast enhancement duration: $\mathrm{AUC}=\mathrm{COAD}>$ normal $>$ CUC. Confocal fluorescence microscopy confirmed that FeDM-FITC-SLNs were mainly distributed in the intestinal mucosa and tumor capsule.

Conclusion: Iron-based SLNs are promising alternatives for contrast enhancement at T1-weighted MRI and will help in the differential diagnosis of intestinal bowel diseases (IBDs).

Keywords: solid lipid nanoparticles, magnetic resonance imaging, contrast agent, ferric iron, intestinal bowel diseases

\section{Introduction}

Intestinal bowel diseases (IBDs), including Crohn's disease and ulcerative colitis, are destructive and recurring tissue disorders that involve dysregulation of the local immune response and compromised intestinal barrier function. ${ }^{1}$ Some studies have shown that the incidence of colitis-associated cancer (CAC) is closely associated with ulcerative colitis. ${ }^{2}$ Colorectal cancer (CRC) refers to malignant lesions of the colonic epithelium that develop due to various environmental or genetic carcinogenic factors. ${ }^{3}$ The incidence and mortality rate of CRC are the third and second among all malignant tumors, respectively. ${ }^{4}$ Although colonoscopy is widely used for colorectal cancer screening, it still suffers from relatively low sensitivity and specificity for adenomas. ${ }^{5}$ Furthermore, screening compliance remains low due to the invasive and unpleasant nature of colonoscopy as well as its relatively high cost. ${ }^{6}$

Received: 12 November 202 Accepted: 4 February 2022

Published: 22 February 2022
International Journal of Nanomedicine 2022:|7 82|-836

821

(c) (7) () 2022 Wang et al. This work is published and licensed by Dove Medical Press Limited. The full terms of this license are available at https://www.dovepress.com/terms. (c) ${ }_{\mathrm{BY}} \mathrm{NC}$ php and incorporate the Creative Commons Attribution - Non Commercial (unported, v3.0) License (http://creativecommons.org/licenses/by-n//3.0/). By accessing the work you hereby accept the Terms. Non-commercial uses of the work are permitted without any further permission from Dove Medical Press Limited, provided the work is properly attributed. For permission for commercial use of this work, please see paragraphs 4.2 and 5 of our Terms (https://www.dovepress.com/terms.php). 
The main advantage of MR colonography is the high soft tissue contrast and the use of nonionizing radiation. ${ }^{7,8}$ Current MR colonography is based on an intravenous injection of a $\mathrm{Gd}^{3+}$-complex to enhance the colorectal walls along with an enema administration of negative contrast agent such as water, carbon dioxide, or room air for colorectal distension. ${ }^{9,10}$ However, gadolinium-based contrast agents (GBCA) were associated with a devastating and potentially fatal condition called nephrogenic systemic fibrosis (NSF) in 2006. ${ }^{11,12}$ This concern over the long-term safety of GBCA also motivates the search for less toxic and more effective alternatives. ${ }^{13}$

Trivalent iron is another paramagnetic metal ion with five unpaired electrons and a high longitudinal relaxivity ${ }^{14}$ It also has low biotoxicity as a normal trace element in the body - the body contains approximately $4 \mathrm{~g}$ of iron and has highly regulated systems for its safe transport and storage. ${ }^{15}$ An increasing number of scholars have been focused on the study of Fe-based contrast agents. Nevertheless, not all iron-containing compounds can function as T1 MRI contrast agents. Some studies suggest that iron-based contrast agents undergo $\mathrm{T}_{1}$ shortening effects to create tissue contrast when $\mathrm{Fe}^{3+}$ cations are incorporated with catechol ligands. ${ }^{16}$ In this study, the small molecule L-dopa methyl ester hydrochloride was used as a catechol ligand. ${ }^{17} \mathrm{Fe}^{3+}$ and L-dopa methyl ester hydrochloride complexes were successfully synthesized and defined as FeDM.

Intestinal wall-absorbable solid lipid nanoparticles (SLNs) are drug delivery systems that can reduce the risk of contrast agents. Unlike systemic administration, the rectal route offers a noninvasive and useful route for drug administration and offers a relatively stable environment for drug delivery, thus leading to a constant and steady-state concentration of drug in the local intestinal wall. ${ }^{18}$ However, poor gastrointestinal permeability due to the epithelial cell monolayer significantly affects the enema bioavailability of numerous drugs. ${ }^{19}$ SLNs have been extensively investigated and developed as a potential nanocarrier for digestive tract drug delivery across intestinal epithelial cell monolayers. ${ }^{20}$ Gadolinium-diethylenetriaminepentaacetic acid (Gd-DTPA) is one of the most commonly used clinical GBCA. Our previous work synthesized SLNs loaded with Gd-DTPA as an MR colonography contrast agent to enhance the colorectal walls or tumors via direct uptake by the colorectum. ${ }^{21-23}$

Here, FeDM- and FITC-loaded SLNs were developed as an intestinal wall-absorbable contrast agent for the detection of intestinal lesions. We then compared their $\mathrm{T}_{1}$ contrast effects with a commercial GBCA and evaluated their value in MR imaging. We further studied whether this approach could assist in differential diagnosis of intestinal bowel diseases based on the different absorptive capacity of SLNs in different bowel conditions. ${ }^{24}$

\section{Materials and Methods}

\section{Materials}

Levodopa methyl ester hydrochloride and N-((2-chloro-3-((phenylimino)methyl)cyclohex-2-en-1-ylidene)methyl)aniline hydrochloride were acquired from Shanghai Bide Pharmatech Ltd., Shanghai, China. Octadecylamine (ODA) was from Fluka, UK. Span-80, Tween-80, anhydrous sodium acetate, ethanol, ether, sodium hydroxide, and ferric nitrate were purchased from Sinopharm Chemical Reagent Co., Ltd., Shanghai, China. The n-hexane was purchased from Shanghai Titan Scientific Co., Ltd., Shanghai, China. Glyceryl monostearate was purchased from Shanghai Macklin Biochemical Co., Ltd., Shanghai, China. Gd-DTPA was a gift from Beilu Pharmaceutical Co., Ltd., Beijing, China. Fluorescein isothiocyanate (FITC) and 3-ethyl-2-methylbenzothiazolium iodide was from Shanghai Aladdin Biochemical Technology Co., Ltd. Deuterated chloroform $\left(\mathrm{CDCl}_{3}\right)$ was from Cambridge Isotope Laboratories, Inc., UK. Hexafluoroisopropanol (HFIP) was purchased from Energy Chemical, Shanghai, China.

CT-26 cells were purchased from the Cell Bank of the Chinese Academy of Sciences, Shanghai, China. Fetal bovine serum (FBS) was purchased from Gibco, Australia. Penicillin, streptomycin, trypsin, and ethylenediaminetetraacetic acid (EDTA) were purchased from Solarbio, USA. The cell counting kit-8 (CCK-8) was purchased from Dojindo Laboratories, Japan. LysoTracker Red DND-99 and Hoechst 33342 were obtained from Meilun Biotech Co., Ltd., Dalian, China. Azoxymethane (AOM) was from Sigma-Aldrich, St. Louis, MO, USA. Dextran sodium sulphate (DSS) was from Beijing Bitab Biotech Co., Ltd., Beijing, China. Chloral hydrate was purchased from China National Pharmaceutical Group Co., Ltd., Beijing, China. The tissue embedding agent (OCT) was from Solarbio Co., Ltd., Shanghai, China. Formalin was purchased from Zhongshan Cannice Biotechnology Co., Ltd., Zhongshan, China. 


\section{Synthesis of FeDM-FITC-SLNs}

FITC-labeled ODA (ODA-FITC) was synthesized as a fluorescent marker according to our previously reported protocol. ${ }^{25-28}$ Here, $0.9 \mathrm{~mL}$ of $1 \mathrm{M}$ sodium hydroxide aqueous solution and $3 \mathrm{~mL}$ of $100 \mathrm{mM}$ ferric nitrate aqueous solution were rapidly and concurrently added to $3 \mathrm{~mL}$ of a 100-mM DM aqueous solution in an ice bath. Another $3 \mathrm{~mL}$ of water was added after vigorous stirring for $30 \mathrm{~min}$. The FeDM solution was then transferred into a flask. Next, $3 \mathrm{~mL}$ of dry ethanol solution with $18 \mathrm{mg}$ of ODA was added to the flask, and the mixture was stirred at $100 \mathrm{rpm}$ using an electric mixer for $30 \mathrm{~min}$ under $60^{\circ} \mathrm{C}$. Next, $3 \mathrm{~mL}$ of dry ethanol solution with $100 \mathrm{mg}$ of monostearin and $10 \mathrm{mg}$ of ODA-FITC were added. The mixture was heated in a $60^{\circ} \mathrm{C}$ bath (FeDM solution). Then, $54 \mathrm{mg}$ of Tween- 80 dissolved in $3 \mathrm{~mL}$ of water as the aqueous phase was mixed with the oil phase containing $30 \mathrm{~mL}$ of $\mathrm{n}$-hexane and $300 \mathrm{mg}$ of Span- 80 . The homogeneous pre-emulsion was formed by mixing, shaking, and ultrasonic treatment. The FeDM solution was injected into the pre-emulsion and stirred for $5 \mathrm{~min}$ at room temperature. After centrifugation at $9800 \mathrm{rpm}$ for $20 \mathrm{~min}$, the aqueous phase was washed with $\mathrm{n}$-hexane and redispersed in $10 \mathrm{~mL}$ of water containing $2.5 \%$ trehalose dihydrate. FeDM-SLNs were obtained by lyophilization of the aqueous phase.

\section{Characterization of FeDM-FITC-SLNs}

The diameter and morphology of the SLNs were investigated via transmission electron microscopy (TEM, Philips CM12). The average hydrodynamic sizes of the SLNs were measured by dynamic light scattering (DLS) (Malvern Zetasizer Nano ZS, Malvern, UK).

The critical micelle concentration (CMC) of SLNs was characterized by diluting the SLN solution gradually from $0.08 \% \mathrm{w} / \mathrm{w}$ to $0.01 \% \mathrm{w} / \mathrm{w}$. The UV/Vis spectra were measured after dilution, and the peak corresponding to the NIR dye was recorded. The exact concentration was then calculated as the CMC of the SLN when the wavelength of the NIR peak shifted from $730 \mathrm{~nm}$ to $630 \mathrm{~nm}$.

The average encapsulation efficiency is a surrogate for entrapment efficiency. The drug loading efficiency represented the loading capacity of Fe. To evaluate the entrapment efficiency and loading capacity of Fe into SLNs, the resulting FeDM-FITC-SLN samples were dissolved in 1.0 of $\mathrm{mL}$ distilled water and dialyzed versus a cellulose membrane with a molecular weight cutoff of $3.5 \mathrm{~kg} / \mathrm{mol}$. Next, $2 \mathrm{~mL}$ of nitric acid was added, and the solvent was removed at $120^{\circ} \mathrm{C}$. The residue was dissolved in nitric acid (5\%). An atomic absorption spectrophotometer (AAS) (Agilent Technologies, USA) was used to measure the concentrations of $\mathrm{Fe}^{3+}$. The entrapment efficiency (EE) and loading capacity (LC) were calculated via Eqs. 1 and 2, respectively.

$$
\begin{gathered}
\mathrm{EE}(\%)=\frac{\mathbf{m A} \times \mathbf{m C}}{\mathbf{m B} \times \mathbf{m b}} \times 100 \% \\
\mathrm{LC}(\%)=\frac{\mathbf{m A}}{\mathbf{m B}} \times 100 \%
\end{gathered}
$$

Here, $\mathrm{A}$ is the mass of $\mathrm{Fe}$ in the precipitate after centrifuging, $\mathrm{B}$ is the mass of added FeDM-FITC-SLNs, $\mathrm{C}$ is the mass of FeDM-FITC-SLNs after freeze-drying, and D is the mass of Fe added in synthesis.

AAS was used to measure $\mathrm{Fe}^{3+}$, and the two synthesized nanomagnetic particles of FeDM and FeDM-FITC-SLN were prepared by diluting them in $1 \mathrm{~mL}$ of water with an $\mathrm{Fe}^{3+}$ concentration of $0.25,0.5,1.0,2.0$, and $4.0 \mathrm{mM}$. The T1 relaxation time was determined on a 3-T clinical MRI scanner (SIGNA HD, General Electric Healthcare, Milwaukee, WI, USA) at room temperature at varying inversion times ( $\mathrm{TI}=100,200,300,400$, and $600 \mathrm{~ms}$ ), with a fixed repetition time at $\mathrm{TR}=4000 \mathrm{~ms}$. In addition, the imaging sequence of $\mathrm{T} 1$ mapping was used to measure the longitudinal relaxation time (T1). The relaxation rate $\mathrm{r} 1(=1 / \mathrm{T} 1)$ was then calculated at various $\mathrm{Fe}^{3+}$ concentrations.

\section{Cell Culture}

The mouse-derived colon adenocarcinoma cell line CT-26 was cultivated in proliferation medium containing $50 \%$ dulbecco's modified eagle medium (DMEM) high glucose consisting of 10\% FBS and 1\% penicillin/streptomycin under standard culture conditions $\left(37^{\circ} \mathrm{C}, 5 \% \mathrm{CO}_{2}\right)$. 


\section{In vitro Cytotoxicity Assay}

Cell proliferation was measured using the CCK-8 (Dojindo, Japan). First, $4 \times 10^{3}$ CT-26 cells/well were seeded into a 96well plate and incubated overnight at $37^{\circ} \mathrm{C}$. The culture medium was replaced with a medium containing Gd-FITC-SLNs to a final concentration of 0 to $800 \mu \mathrm{g} / \mathrm{mL}$. The CCK- $8(20 \mu \mathrm{L}, 5 \mathrm{mg} / \mathrm{mL})$ was added after $24 \mathrm{~h}, 48 \mathrm{~h}$, and $72 \mathrm{~h}$ of incubation at $37^{\circ} \mathrm{C}$ in a $5 \% \mathrm{CO}_{2}$ atmosphere. The absorbance at $450 \mathrm{~nm}$ was measured to monitor cell proliferation after $2 \mathrm{~h}$.

\section{In vivo Toxicity Assay}

This study was approved by the Animal Welfare and Ethics Review Board of Sir Run Run Shaw Hospital, College of Medicine, Zhejiang University (number SRRSH2020201). All animal experiments were performed in accordance with the guidelines of the Institutional Animal Care and Use Committee (IACUC) of Zhejiang University.

The $20 \mathrm{C} 57 \mathrm{BL} / 6$ mice were randomly divided into a control group and an experimental group (10 mice for each group). In the experimental groups, the mice received a single enema of FeDM-FITC-SLNs $(0.5 \mathrm{~mL}, 250 \mathrm{mg} / \mathrm{mL})$, and the mice in the control group were given the same volume of water. All mice were observed for 14 days for signs of toxicity, their body weights were measured daily, and their mortality was recorded. Finally, the mice were sacrificed and dissected for pathological examination and serum chemistry tests.

\section{In vitro Cellular Uptake Assay}

Cellular uptake was assessed by confocal laser scanning microscopy (CLSM). First, $2 \times 10^{5}$ CT-26 cells/well were seeded into 24-well tissue culture plates in DMEM medium with $10 \%$ fetal bovine serum (FBS) and incubated overnight at $37^{\circ} \mathrm{C}$ to allow the cells to attach. The next day, the media was removed and replaced with media containing $800 \mu \mathrm{g} / \mathrm{mL}$ FeDMFITC-SLNs. The cells were then incubated at $37^{\circ} \mathrm{C}$ and $5 \% \mathrm{CO}_{2}$. After $24 \mathrm{~h}$, cells were rinsed with phosphate buffered saline (PBS) before incubation with LysoTracker (LysoTracker Red DND-99) diluted 1:2000 in DMEM at $37^{\circ} \mathrm{C}$ for 30 min. These cells were then fixed with $4 \%$ paraformaldehyde for $5 \mathrm{~min}$. The CT-26 cells were then stained with $1 \mathrm{mg} / \mathrm{mL}$ Hoechst 33342 for $3 \mathrm{~min}$ at room temperature in the dark and stored at $4^{\circ} \mathrm{C}$ until imaging. Finally, the cells were rinsed again and imaged by CLSM (Zeiss LSM710, Germany).

\section{Animal Modelling}

To investigate whether using FeDM-FITC-SLNs can distinguish the different intestinal diseases in MR colonography, we used three mouse models of intestinal lesions: acute ulcerative colitis (AUC), chronic ulcerative colitis (CUC), and colorectal adenocarcinoma (COAD). In contrast to BALB/c mice, C57BL/6 mice can easily develop chronic inflammation subsequent to the acute response ${ }^{29}$. Thus, all mice used in this study were wild-type C57/B6 female mice.

In 1990, Okayasu et al described a model in which mice receiving oral DSS developed acute and chronic colitis resembling ulcerative colitis (UC). ${ }^{30}$ The use of DSS following prior administration of the mutagen AOM expedites the process of tumor development from several months to as little as 8-10 weeks, thus leading to widespread acceptance of the AOM/DSS model for CAC ${ }^{31}$. Thus, experimental colitis and colitis-associated colorectal carcinogenesis mouse models were chemically induced by the AOM/DSS technique.

Female C57BL/6 mice aged 8 weeks (Vitalriver Co., Ltd., Beijing, China; $\mathrm{n}=80$ ) were randomly allocated to four groups ( $\mathrm{n}=20$ each, body weight $20.4 \pm 1.2 \mathrm{~g}$ ). The mice were housed under $12 \mathrm{~h}$ day/night cycles in a clean grade vivarium at a temperature of $20-25^{\circ} \mathrm{C}$ and a humidity of $55-60 \%$. They had ad libitum access to food and water, as well as adaptable feeding, for one week.

In Group 1, AUC was induced by giving mice 2.5\% DSS (36-50 kg/mol, Beijing Bitab Biotech Co., Ltd., Beijing, China) in their drinking water for one week. Group 2 mice were provided with drinking water containing $2.5 \%$ DSS for 7 days, followed by normal drinking water for 14 days; there were then three more $2.5 \%$ DSS treatment cycles. In Group 3, nine-week-old C57/BL mice were intraperitoneally injected with $10 \mathrm{mg} / \mathrm{kg}$ body weight of AOM (SigmaAldrich, St. Louis, MO, USA). After seven days, 2.5\% DSS was given in the drinking water for 7 days followed by 
14 days of regular drinking water. Then another 7-day cycle of 2.5\% DSS followed by a second two -week rest period was given, followed by a final 7 -day cycle of $2.5 \%$ DSS. Group 4 mice had normal drinking water throughout.

\section{MRI Protocol}

All scans were acquired using a 3.0-T magnetic resonance scanner (SignaHDxt, GE Medical Systems, Milwaukee, WI, USA) equipped with a specialized 1-inch quadrature mouse coil (Shanghai Chenguang Medical Technologies Co., Ltd.). The mice were positioned head first and prone so that the colons were aligned approximately with the center of the RF coil. A $\mathrm{T}_{1}$-weighted SE sequence was used during MR colonography with the following parameters: TR/TE, 520/15 ms; matrix, $256 \times 256$; section thickness, $0.8 \mathrm{~mm}$; spacing, $0.5 \mathrm{~mm}$; Nex, 2; and FOV, $7 \times 7 \mathrm{~cm}$.

\section{In vivo MR Imaging}

To account for tumor heterogeneity, we compared the T1 contrast effects of FeDM-FITC-SLNs with Gd-DTPA in the same tumors at subsequent examinations on two successive days. The Gd complexes were completely eliminated after 24 $\mathrm{h}$ if the glomerular filtration was not diminished. ${ }^{32}$ All mice were then first imaged with Gd-DTPA and then with FeDMFITC-SLNs one day later. On the first day, all mice were anesthetized via an intraperitoneal injection of $4 \%$ chloral hydrate (China National Pharmaceutical Group Co., Ltd., Beijing, China). To better image the intestinal architecture, $0.5 \mathrm{~mL}$ of room air was infused into the colorectum through a 1-mL syringe and a 24-gauge cannula. The anus was ligated with a cotton thread. Baseline MRI scans were then collected. After completing the MRI scans, each mouse was removed from the coil for an injection of Gd-DTPA $(0.1 \mathrm{mmol} / \mathrm{kg})$ and placed back into the coil. MRI examinations were performed at 0, 20, 40, 60, 80, and 100 min following Gd-DTPA injection.

One day later, each experiment was repeated on each mouse including aesthesia, intestinal inflation, and baseline MR colonography. Each mouse was then removed from the coil for a liquid enema of FeDM-FITC-SLNs $(250 \mathrm{mg} / \mathrm{mL}$, $0.5 \mathrm{~mL}$ ) and placed in a cage for $20 \mathrm{~min}$. Leakage from the rectum was prevented by suturing the anus. After $20 \mathrm{~min}$, the intestines were rinsed with saline and blotted dry before repeat intestinal inflation. We finally performed serial MRI examinations from 0 to 100 min after a 20-min FeDM-FITC-SLNs retention enema.

\section{Imaging Analysis}

The images were analyzed with ADVANCE 4.3 Workstation software (General Electric Medical Systems). Image noise was defined as the SD of signal intensities measured in the background of each image. The mean signal intensity (SI) of each sample was measured in regions-of-interest (ROIs). The SNR of each image was calculated to quantitatively comparing the measurements between different time points and between different intestinal walls or lesions $(\mathrm{SNR}=\mathrm{SI}$ enhanced area/SD noise).

\section{Histologic Analysis}

The mice were euthanized in a $\mathrm{CO}_{2}$ chamber after MR colonography, and the intestinal lesions were harvested. Each tissue was divided into two parts: The first portion used dual-fluorescence staining and confocal microscopic analysis, and the second one was processed for histology. The first portion of fresh tissue was incubated at $37^{\circ} \mathrm{C}$ for cell membrane staining with LysoTracker Red DND-99 for $30 \mathrm{~min}$. The sample was then rinsed three times with PBS. Samples were then brought to $-80^{\circ} \mathrm{C}$, sectioned on a 5 - $\mu \mathrm{m}$-thick cryostat microtome (HM550, Microm), and thawmounted on covered glass slides. The sections were stained with Hoechst-33342 $(1 \mathrm{mg} / \mathrm{mL})$ for three minutes and rinsed with PBS. Finally, histological sections were then examined by CLSM. The second portion of each tissue was sectioned after fixing in $10 \%$ buffered formalin for $24 \mathrm{~h}$. These sections were stained with H\&E for histopathological analysis.

\section{Statistical Analysis}

All results were reported as the mean \pm standard deviation (SD). One-way analysis of variance was used to compare between groups and within the same group at different time points using GraphPad Prism 8.3.0 (GraphPad Software, Inc., La Jolla, California, USA). A P-value of $<0.05$ was considered statistically significant. 


\section{Results}

\section{Characterization of FeDM-FITC-SLNs}

We successfully synthesized FeDM-FITC-SLNs using the solvent diffusion method (Figure 1A). Transmission electron microscopy (TEM) showed that the nanoparticles were spherical with a mean diameter of $100 \mathrm{~nm}$ (Figure 2A and Figure B). DLS revealed that FeDM-FITC-SLNs had a broad range in size with a mean diameter of $220 \mathrm{~nm}$ (Figure 2C). The CMC of FeDM-FITC-SLNs was $0.04 \%(\mathrm{w} / \mathrm{w})$ as measured by fluorescence probe spectrometry. The AAS results show that the encapsulation and drug loading efficiencies were $60.3 \%$ and $94.3 \%$, respectively. $\mathrm{A}_{1}$ map showed that the $\mathrm{T}_{1}$ values increase with increasing $\mathrm{Fe}^{3+}$ concentrations (Figure 2D). Moreover, the relaxivities of FeDM $\left(\mathrm{r}_{1}=3.42\right.$ $\left.\mathrm{mM}^{-1} \cdot \mathrm{s}^{-1}\right)$ and FeDM-FITC-SLNs $\left(\mathrm{r}_{1}=4.02 \mathrm{mM}^{-1} \cdot \mathrm{s}^{-1}\right)$ were similar to the clinical contrast agent Gd-DTPA $\left(\mathrm{r}_{1}=4.11\right.$ $\mathrm{mM}^{-1} \cdot \mathrm{s}^{-1}$ ) (Figure 2E). ${ }^{33}$

\section{Cytotoxicity of FeDM-FITC-SLNs}

Toxicity evaluation used a CCK-8 assay to assess the effects of FeDM-FITC-SLNs on cell viability. Over 90\% of cell viability was maintained at a final concentration of $0-800 \mu \mathrm{g} / \mathrm{mL}$ for $24 \mathrm{~h}, 48 \mathrm{~h}$, and $72 \mathrm{~h}$ (Figure 3A). Neither agent exerted significant toxic effects on cells.

\section{In vivo Toxicity of FeDM-FITC-SLNs}

No mortality was observed in any group for two weeks. The body weight of both groups increased (Table 1). There were no abnormalities in the histopathological examination of major organs (Figure 3B). Serum biochemistry variables were measured in all mice including alanine aminotransferase (ALT), aspartate transaminase (AST), creatinine (CR), and blood urea nitrogen (BUN). All samples were within normal range with no significant group differences (Table 2).

\section{In vitro Cellular Uptake of FeDM-FITC-SLNs}

We noted fluorescent signals under a confocal microscope and found green fluorescent signals that originated from FeDM-FITC-SLNs in the cytoplasm. Co-localization of red and green fluorescence was visualized by the yellow fluorescence appearing after merging both signals. Most green fluorescence signals overlapped with red fluorescence from lysotracker suggesting good overlap between the SLNs and the lysosomes (Figure 4).

\section{In vivo MRI of Mouse Models}

All mice successfully completed the MR study without complications. The signal intensities in the intestinal walls and lesions were immediately enhanced and peaked at $3 \mathrm{~min}$ after injection of Gd-DTPA (Figure 5A). Thereafter, the enhancement began to decrease rapidly with complete recovery to near initial levels at $80 \mathrm{~min}$. All four groups of mice showed no significant difference after Gd-DTPA injection. The signal intensity in the intestinal walls and lesions also increased after FeDM-FITCSLNs enema versus Gd-DTPA. The enhancement was maintained at this level for several minutes (Figure 5B). Importantly, these images indicate that there were rather significant differences between normal tissues and intestinal lesions in terms of the duration of enhancement. The shortest group (CUC) had a duration of up to $\sim 40 \mathrm{~min}$, followed by the second longest group (normal) with a duration of up to $\sim 80 \mathrm{~min}$. The longest groups (AUC and COAD) remained enhanced for up to $\sim 100 \mathrm{~min}$.

The SNR mean values were calculated for the four groups (Figure 6 and Table 3). Figure 6A shows that the SNR increased two-fold immediately after injection of Gd-DTPA. The SNR returned to baseline levels by $100 \mathrm{~min}$. After the FeDM-FITC-SLNs enema, the SNR increased from $20 \mathrm{~min}$ through $100 \mathrm{~min}$. There was a 1.9-fold increase at $20 \mathrm{~min}$, and a 1.5-fold increase at $100 \mathrm{~min}$. In the second group, the SNR increased to 1.8-fold and 1.6-fold after administration of GdDTPA and FeDM-FITC-SLNs, respectively. They both returned to basal levels after $60 \mathrm{~min}$ (Figure 6B). The SNR had the longest increase after FeDM-FITC-SLN enema in the COAD group (Figure 6C). Versus Gd-DTPA, the SNR increased to 1.4-fold even at 100 min post-administration of FeDM-FITC-SLNs. In the normal intestine wall, the SNR increased to 1.8-fold immediately after injection of Gd-DTPA with a decrease through $60 \mathrm{~min}$. A FeDM-FITC-SLN enema extended the duration of signal to $\sim 80 \mathrm{~min}$ from $60 \mathrm{~min}$ (Figure 6D). 


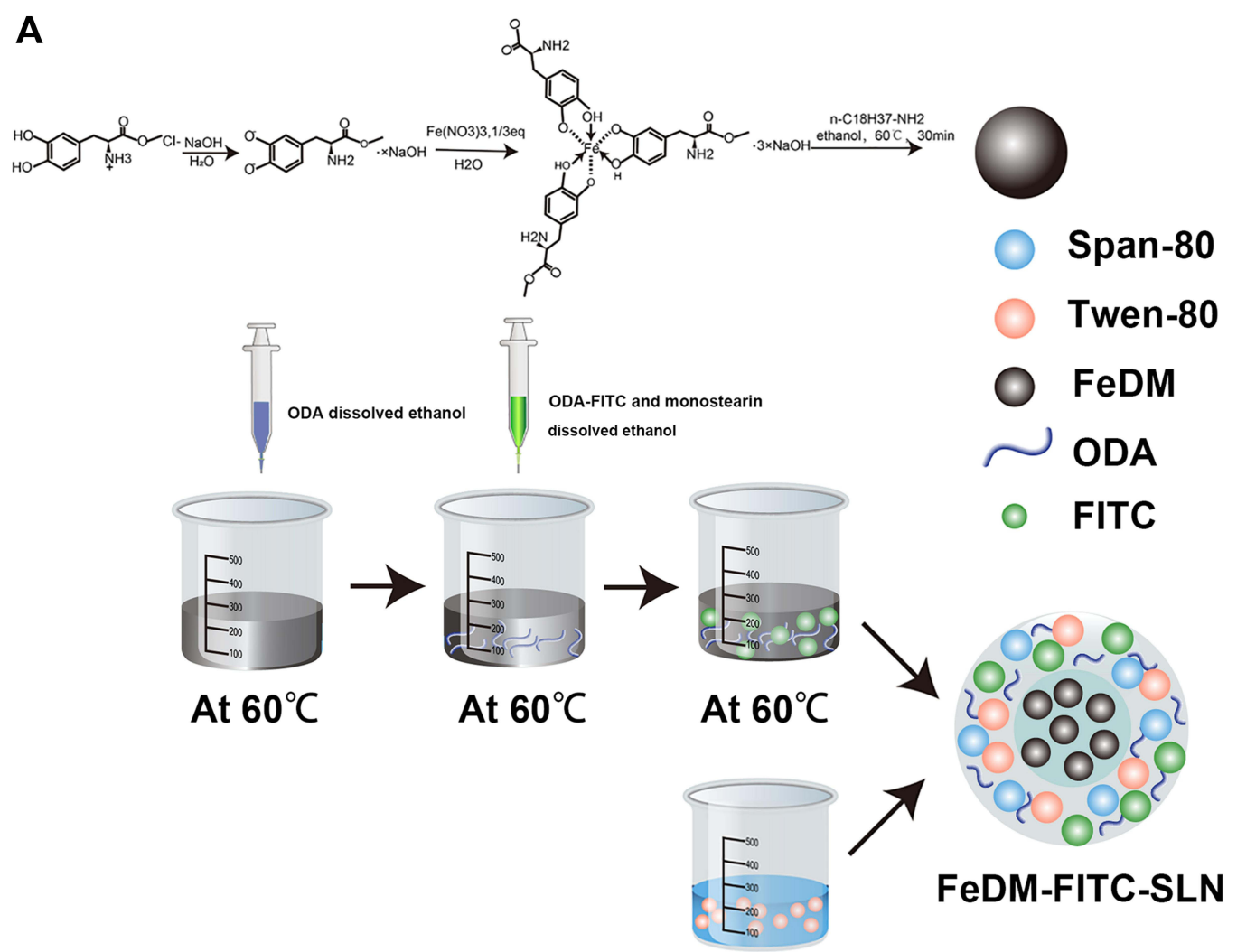

At room temperature

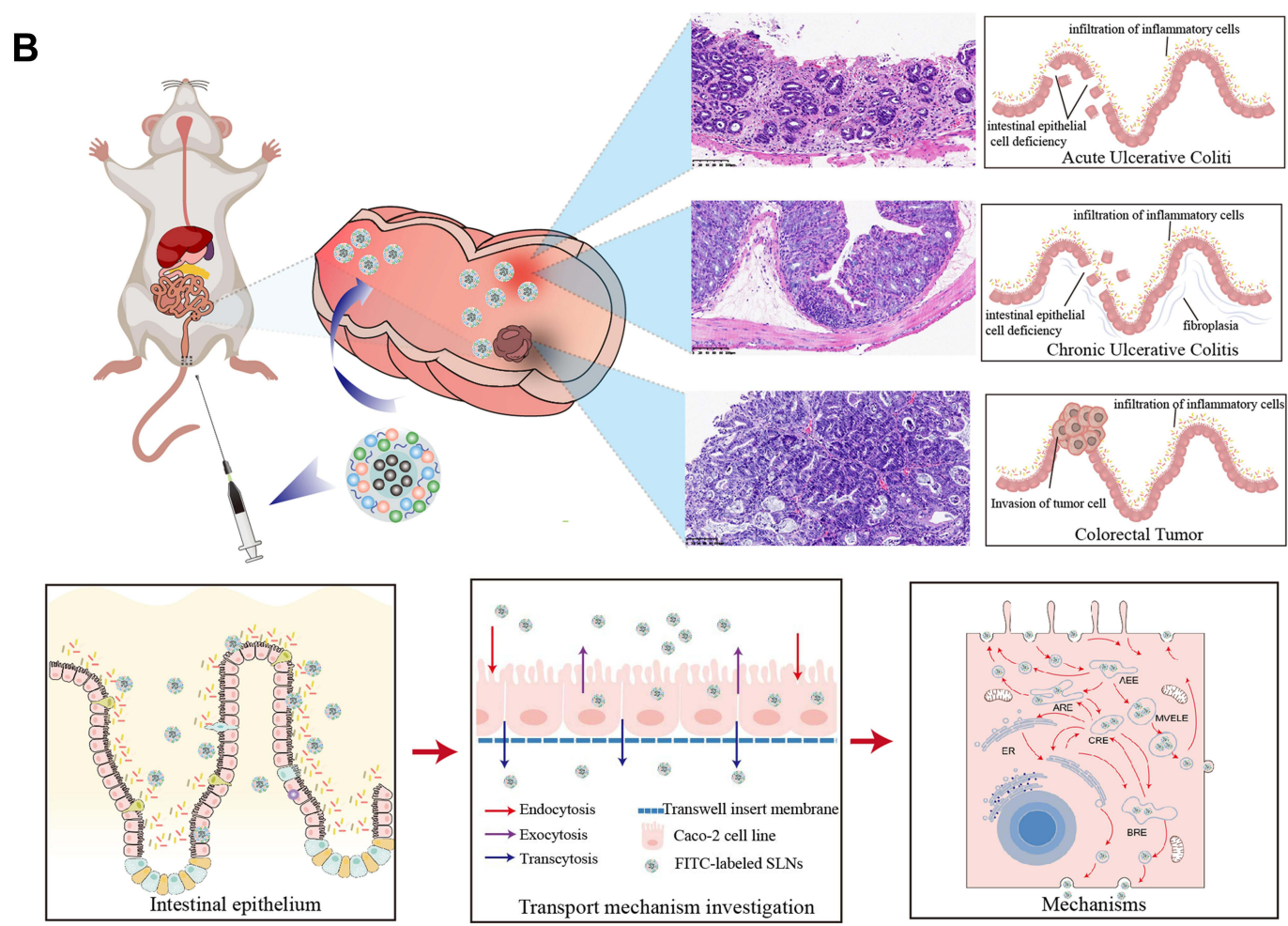

Figure I Schematic illustrations of nanoparticles.

Notes: (A) Schematic illustrations for the synthesis of the FeDM and FeDM-FITC-SLN. (B) Schematic diagram for the absorption and transport of SLNs in different intestinal lesions.

Abbreviations: FeDM, $\mathrm{Fe}^{3+}$ and L-dopa methyl ester hydrochloride complexes; FITC, fluorescein isothiocyanate; SLN, solid lipid nanoparticle; ODA, octadecylamine. 

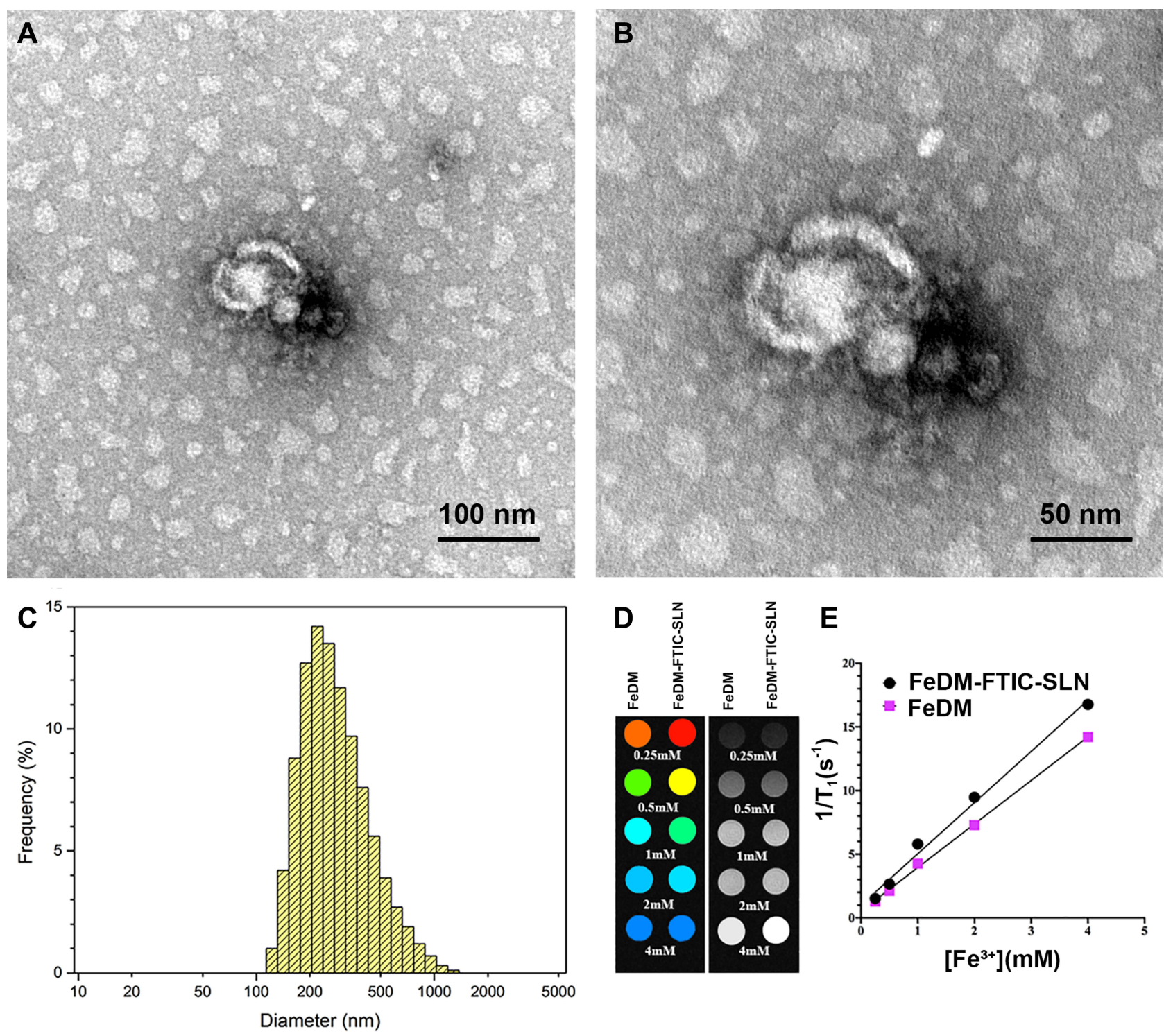

Figure 2 Characterization of nanoparticles.

Notes: (A and B) Transmission electron microscopy images of FeDM-FITC-SLNs. (C) Histogram of nanoparticle size distribution. (D) TI-weighted MR images of FeDM and FeDM-FITC-SLNs solutions at different Fe concentration. (E) Linear fitting of the inverse $T_{1}$ values.

Abbreviations: FeDM, $\mathrm{Fe}^{3+}$ and L-dopa methyl ester hydrochloride complexes; FITC, fluorescein isothiocyanate; SLN, solid lipid nanoparticle.

\section{Histological Assessments}

The third column in Figure 7 shows that the FITC fluorescence signal alone (fourth and fifth columns) overlaps with both lysosomes and FITC signals. The co-localized expression of both appears yellow in CLSM. The yellow fluorescence appeared after merging both channels and was seen in the intestinal mucosa layer or the capsule of the tumors in all three tumor groups.

Histological assessment of HE-stained tissue sections is shown in the sixth column of Figure 7. The first row shows that the intestinal mucosa appeared hyperemic and edematous with mucosal exfoliation and ulceration. Infiltration of subepithelial inflammatory cells was significantly increased suggesting that the disease in the first group resembled human acute ulcerative colitis. In the second group, the intestinal glands were damaged and disorganized and contained necrotic epithelial cells, infiltrated inflammatory cells, and multinucleated giant cells. Stroma deposition and hyperplasia of fibrous tissue were found in the submucosa. Thus, pathological examination revealed chronic inflammation on the rectal mucosa surface. The tumor cells in the third group were disordered with no exact morphological structure but a generally 

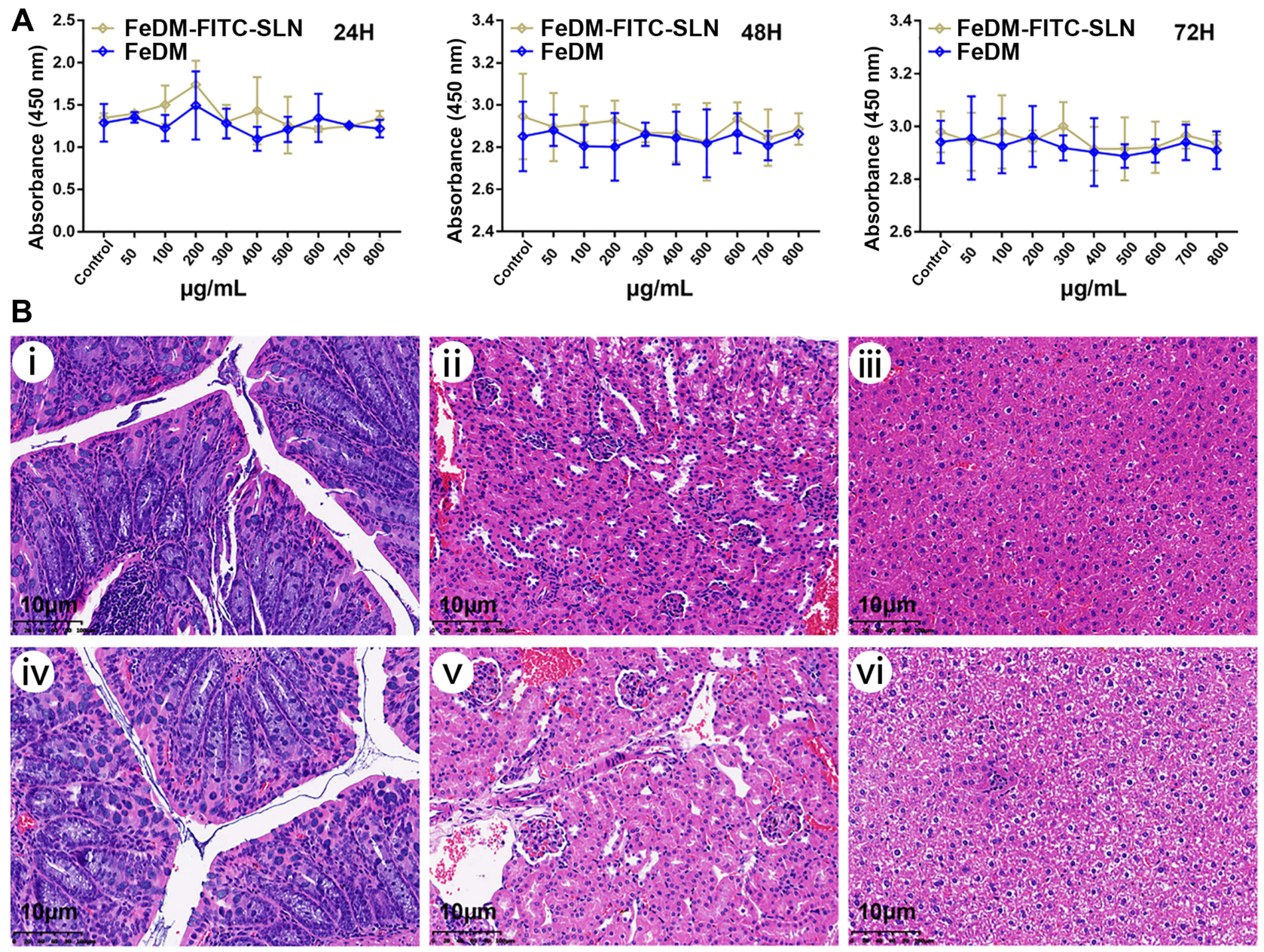

Figure 3 Toxicity assay of nanoparticles.

Notes: (A) CT-26 cells in 96-well plates were treated with different concentrations of FeDM or FeDM-FITC-SLN for different times; cell viability data was determined using the CCK-8 assay. (B) After I4 days, colon (I and IV), kidney (II and V), and liver (III and VI) were harvested and taken for histopathology. Control (I, II, and III) and experimental (IV, $\mathrm{V}$, and $\mathrm{VI}$ ) animals were included.

Abbreviations: FeDM, $\mathrm{Fe}^{3+}$ and L-dopa methyl ester hydrochloride complexes; FITC, fluorescein isothiocyanate; SLN, solid lipid nanoparticle.

round shape. The nuclei were large and abnormally shaped, the chromatins were deeply colored; they were obviously heterogeneous. In the fourth group, HE colon staining showed that the intestinal mucosa epithelium was intact, and that the intestinal gland was composed of lamina propria and a mucosa muscle layer.

Table I Mice Were Observed Daily for Changes in Body Weight for I4 Days (Day I to Day 14)

\begin{tabular}{|l|l|l|l|l|l|l|l|l|}
\hline & Before & DI & D2 & D3 & D4 & D5 & D6 & D7 \\
\hline $\begin{array}{l}\text { Control } \\
\text { SLNs }\end{array}$ & $19.3 \pm 1.0$ & $19.4 \pm 1.2$ & $19.6 \pm 0.7$ & $19.7 \pm 0.5$ & $20.0 \pm 0.5$ & $20.4 \pm 0.8$ & $20.6 \pm 0.6$ & $20.9 \pm 0.8$ \\
& $18.9 \pm 0.9$ & $19.0 \pm 1.2$ & $19.2 \pm 1.3$ & $19.5 \pm 1.1$ & $19.6 \pm 1.0$ & $19.8 \pm 0.7$ & $20.1 \pm 0.5$ & $20.4 \pm 1.0$ \\
\hline & D8 & D9 & D 10 & DII & D 12 & D 13 & D I4 & \\
\hline $\begin{array}{l}\text { Control } \\
\text { SLNs }\end{array}$ & $21.0 \pm 0.9$ & $21.3 \pm 0.9$ & $21.3 \pm 1.0$ & $21.6 \pm 0.9$ & $21.7 \pm 1.1$ & $21.9 \pm 1.0$ & $22.1 \pm 0.7$ & $21.8 \pm 1.0$ \\
\hline
\end{tabular}


Table 2 Effect on Serum Chemistry Profiles After Administration

\begin{tabular}{|l|l|l|l|l|}
\hline & ALT (U/L) & AST (U/L) & CR (nmol/L) & BUN (mmol/L) \\
\hline Control & $104.0 \pm 29.4$ & $143.2 \pm 10.6$ & $36.9 \pm 1.9$ & $10.0 \pm 0.8$ \\
SLNs & $105.5 \pm 20.5$ & $138.9 \pm 10.9$ & $37.1 \pm 12.1$ & $10.8 \pm 1.3$ \\
\hline
\end{tabular}

Abbreviations: ALT, alanine aminotransferase; AST, aspartate aminotransferase; CR, creatinine; BUN, blood urea nitrogen.

\section{Discussion}

Researchers have long sought an alternative to Gd-DTPA. Thus, this study sought to develop iron-loaded SLNs as an absorbable contrast agent to assess the uptake function of the intestinal lesions. Here, FeDM-FITC-SLNs with homogeneously dispersed nanoparticles were prepared via a solvent diffusion method. We further established three animal models of intestinal diseases to compare the different enhancement effects after administration of FeDM-FITC-SLNs or Gd-DTPA. FeDM-FITC-SLNs showed a similar level of contrast enhancement as Gd-DTPA. More importantly, we found that the duration of contrast enhancement led to considerable differences in different intestinal lesions after an enema of FeDM-FITC-SLNs.

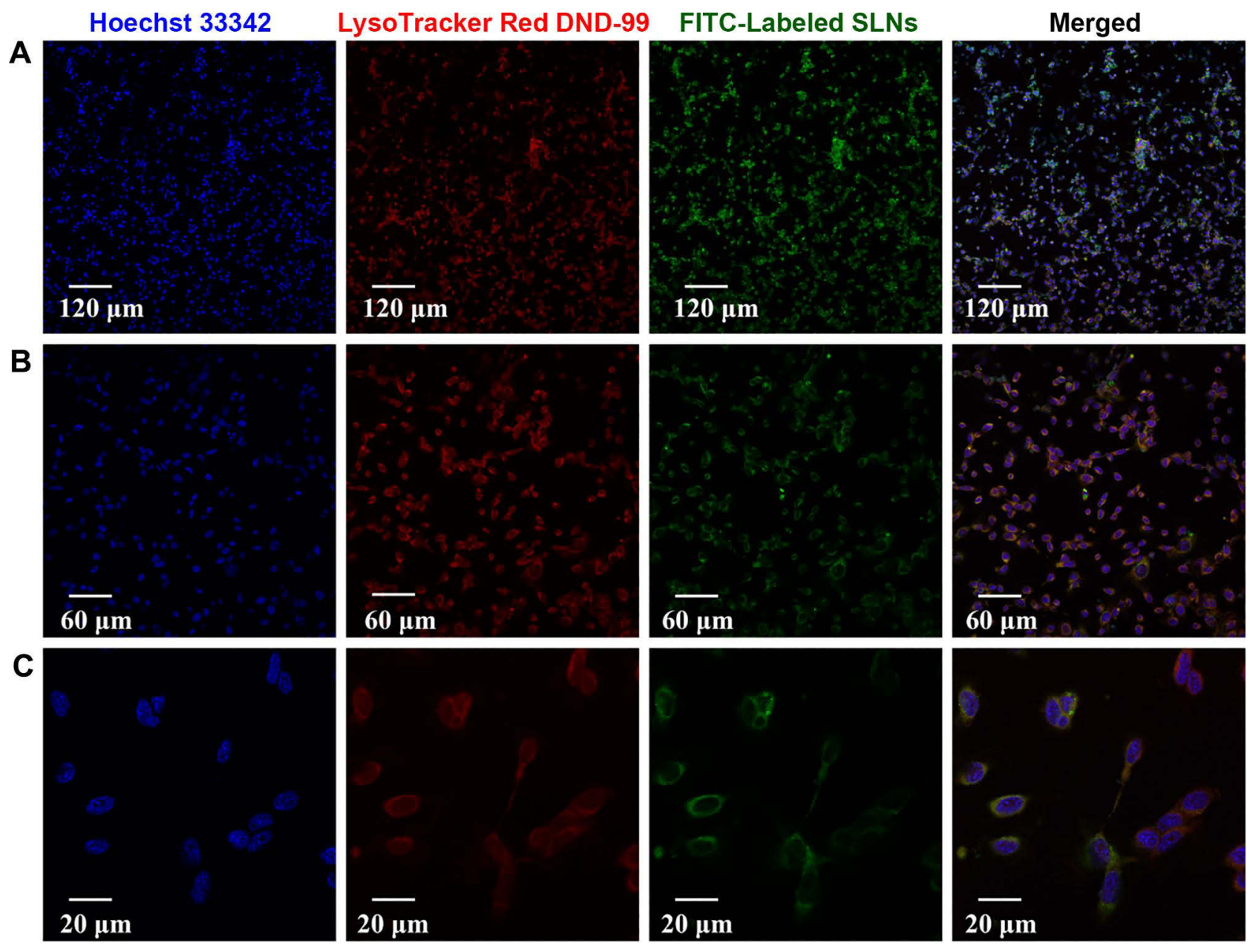

Figure 4 Cellular uptake of nanoparticles in vitro.

Notes: Cells were incubated with $800 \mu \mathrm{g} / \mathrm{mL}$ FeDM-FITC-SLNs at $37^{\circ} \mathrm{C}$ for $24 \mathrm{~h}$ and then stained with Hoechst and LysoTracker Red. Images were captured using confocal microscopy. Blue represents nuclei stained with Hoechst dye; red represents lysosome; green represents FITC-labeled SLNs; and yellow shows colocalization of SLNs and lysosomes. (A) magnification, $\times 100$; (B) magnification, $\times 200$; and (C) magnification, $\times 600$.

Abbreviations: FITC, fluorescein isothiocyanate; SLN, solid lipid nanoparticle. 

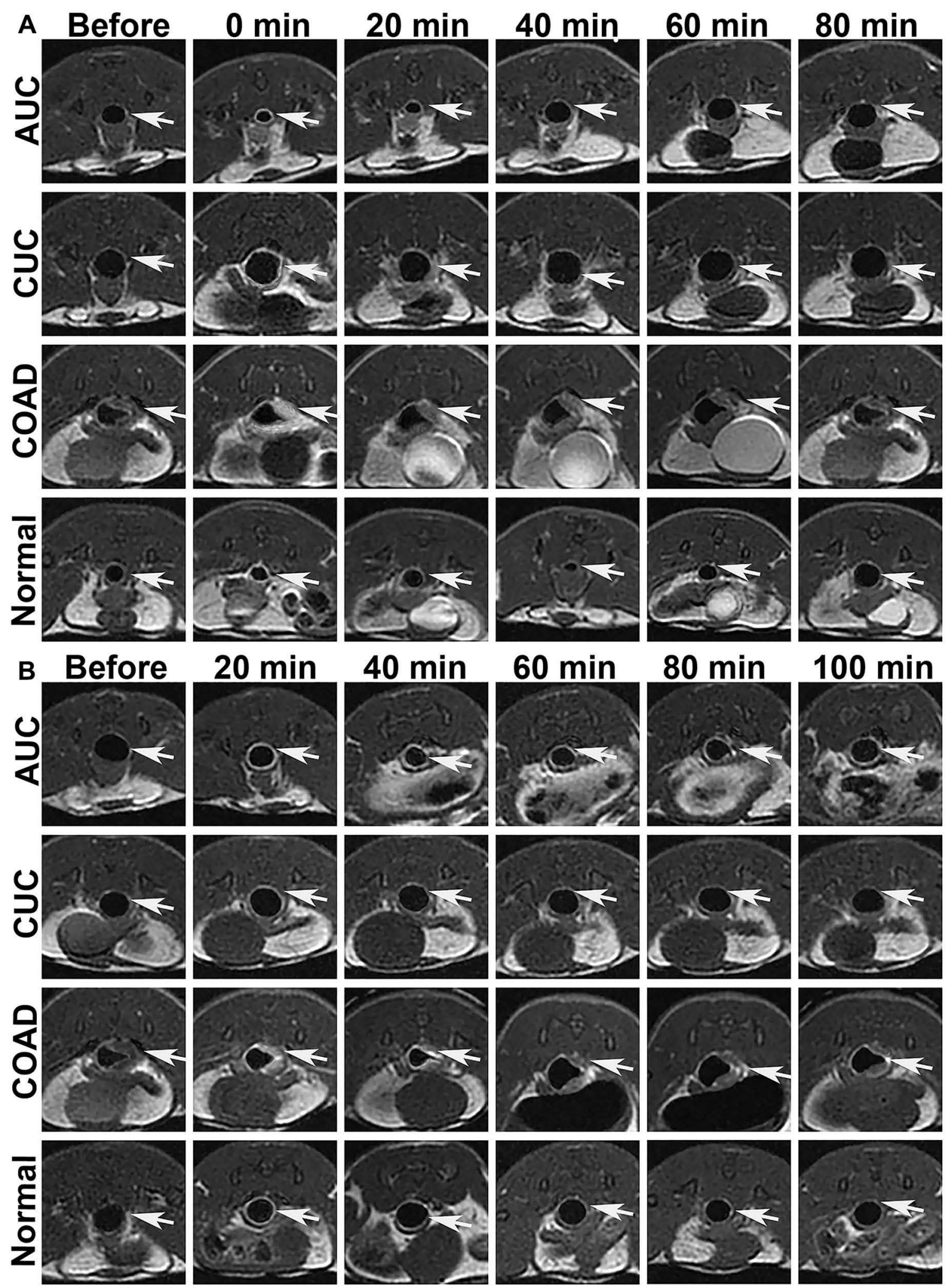

Figure 5 In vivo $M R$ imaging.

Notes: In vivo $T_{1}$-weighted MRI of the experimental mouse models before and after intravenous injection of Gd-DTPA (A) and enema of FeDM-FITC-SLN (B). The white arrows indicate intestines.

Abbreviations: Gd-DTPA, gadolinium-diethylenetriaminepentaacetic acid; $\mathrm{FeDM}, \mathrm{Fe}^{3+}$ and L-dopa methyl ester hydrochloride complexes; FITC, fluorescein isothiocyanate; SLN, solid lipid nanoparticle; AUC, acute ulcerative colitis; CUC, chronic ulcerative colitis; COAD, colon adenocarcinoma. 

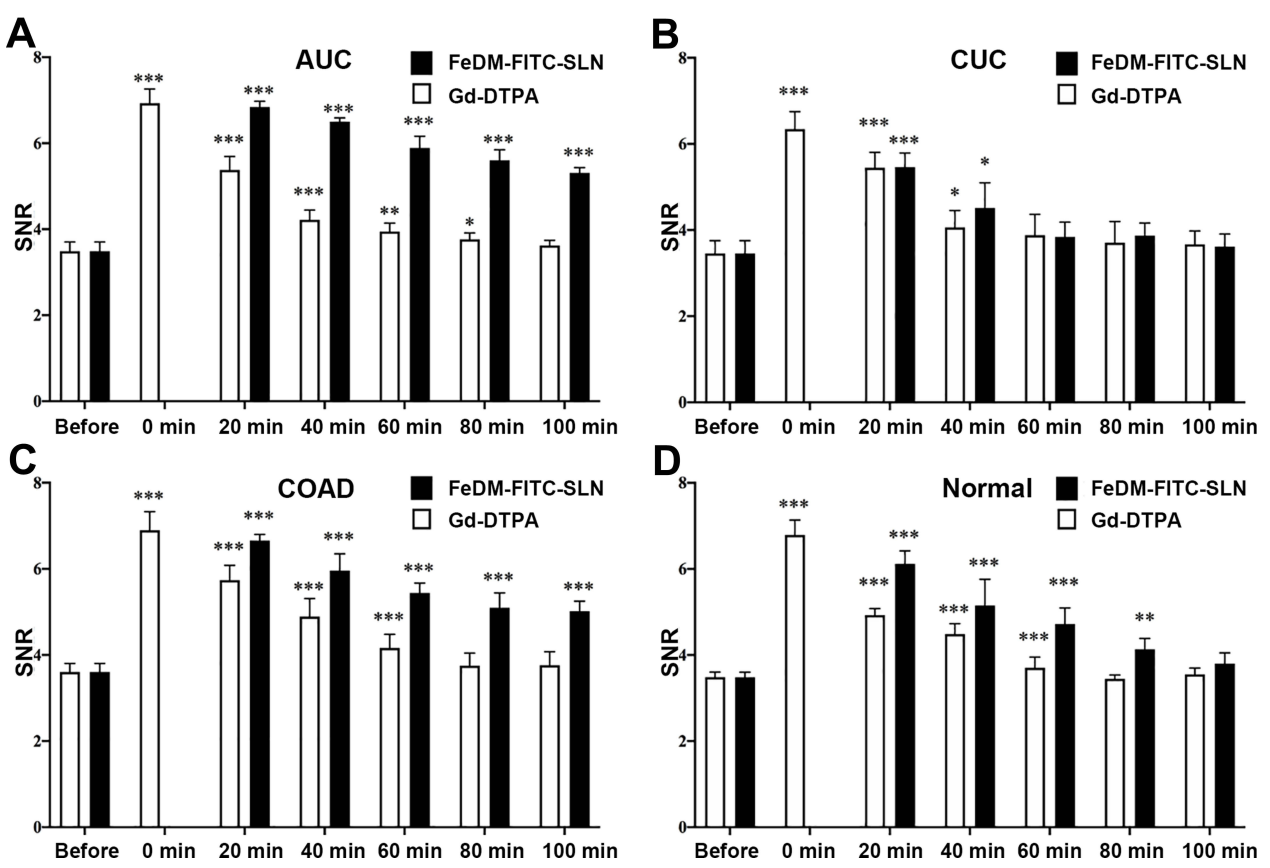

Figure 6 Imaging analysis for MRI.

Notes: MR SNR for the intestine in different mouse models $(\mathbf{A})$ acute ulcerative colitis; (B) chronic ulcerative colitis; (C) colon adenocarcinoma; (D) normal) after intravenous injection of Gd-DTPA and enema of FeDM-FITC-SLN. ${ }^{*} \mathrm{p}<0.05$ vs before; ${ }^{* *} \mathrm{p}<0.01$ vs before; $* * * \mathrm{p}<0.00 \mathrm{I}$ vs before.

Abbreviations: SNR, signal-to-noise ratio; Gd-DTPA, gadolinium-diethylenetriaminepentaacetic acid; FeDM, $\mathrm{Fe}^{3+}$ and L-dopa methyl ester hydrochloride complexes; FITC, fluorescein isothiocyanate; SLN, solid lipid nanoparticle; AUC, acute ulcerative colitis; CUC, chronic ulcerative colitis; COAD, colon adenocarcinoma.

After Gd-DTPA injection, most of malignant intestinal lesions MRI characteristics are "fast forward and fast out." Benign lesions are more likely to be diagnosed as "fast forward and slow out" or "slow forward and slow out." The enhanced modes are helpful in differentiating between benign and malignant intestinal lesions. However, there is a short time window for making a diagnosis because Gd-DTPA is rapidly (within $\sim 1$ hour) cleared from the body. FeDM-FITCSLNs is completely based on the intestinal absorptive function. Histopathological lesion changes will significantly affect the absorption rate and the local concentration of the FeDM-FITC-SLNs (Figure 1B) ${ }^{34,35}$ Thus, FeDM-FITCSLNs offer a dramatically different mode of lesion enhancement. Greater divergence among the lesions was also observed.

Our results showed that FeDM-FITC-SLNs can significantly enhance the bowel wall or tumor after enema administration. The degree of enhancement was slightly higher than that obtained after intravenous Gd-DTPA injection. Second, FeDM-FITC-SLNs in different intestinal bowel lesions caused different modes of enhancement in MR colonography. After enema with FeDM-FITC-SLNs, the MRI showed the following contrast enhancement duration: $\mathrm{AUC}=\mathrm{COAD}>$ normal $>\mathrm{CUC}$. The agent is difficult to retain in the colorectal wall with chronic inflammation. The opposite may be true in AUC. The enhancement effects and duration are significantly longer than those after injection of Gd-DTPA.

For colorectal malignancies, the MRI characteristics seemed to be "fast forward and slow out." Enema with FeDMFITC-SLN can improve the differential diagnostic capacity for intestinal bowel diseases versus Gd-DTPA. In future clinical therapeutic trials, the degree of enhancement could be assessed at $60 \mathrm{~min}$ and $90 \mathrm{~min}$. At the first time point, CUC could be easily diagnosed with FeDM-FITC-SLNs that are then likely to be cleared. At the second time point, we distinguished between normal and acute intestinal walls because the latter retained enhancement. The clear boundary between the tumor and intestinal wall was delineated based on lasting tumor enhancement. Thus, FeDM-FITC-SLNs have a clinically meaningful benefit in reducing the rate of missed diagnosis for colorectal tumors.

Chai et al found that endocytosis of the SLNs was mediated by the macropinocytosis pathway and clathrin- and caveolae (or lipid raft)-related routes. ${ }^{35}$ Here, lysosomes were labeled to investigate the destinations of the transported SLNs. Colocalization of these organelles with the SLNs was then observed using CLSM. ${ }^{36}$ In line with to previous 
Table 3 MR Signal-to-Noise Ratio (SNR) for Intestine in the Different Mouse Models

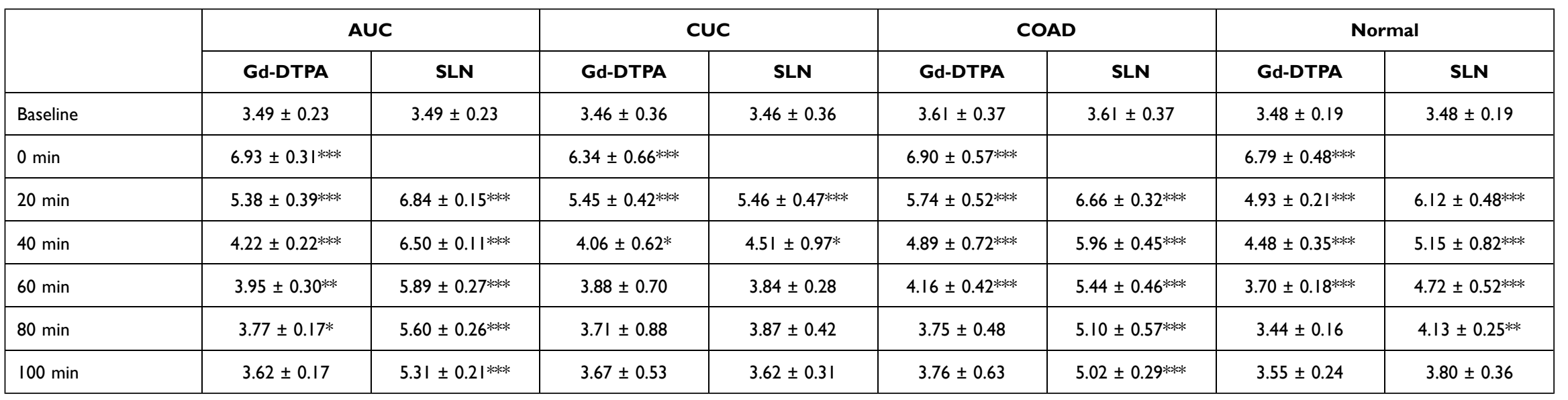

Notes: $*_{\mathrm{p}}<0.05$ vs baseline; ${ }^{* *} \mathrm{p}<0.01$ vs baseline; $* * * \mathrm{p}<0.001$ vs baseline

Abbreviations: Gd-DTPA, gadolinium-diethylenetriaminepentaacetic acid; SLN, solid lipid nanoparticle; AUC, acute ulcerative colitis; CUC, chronic ulcerative colitis; COAD, colon adenocarcinoma. 


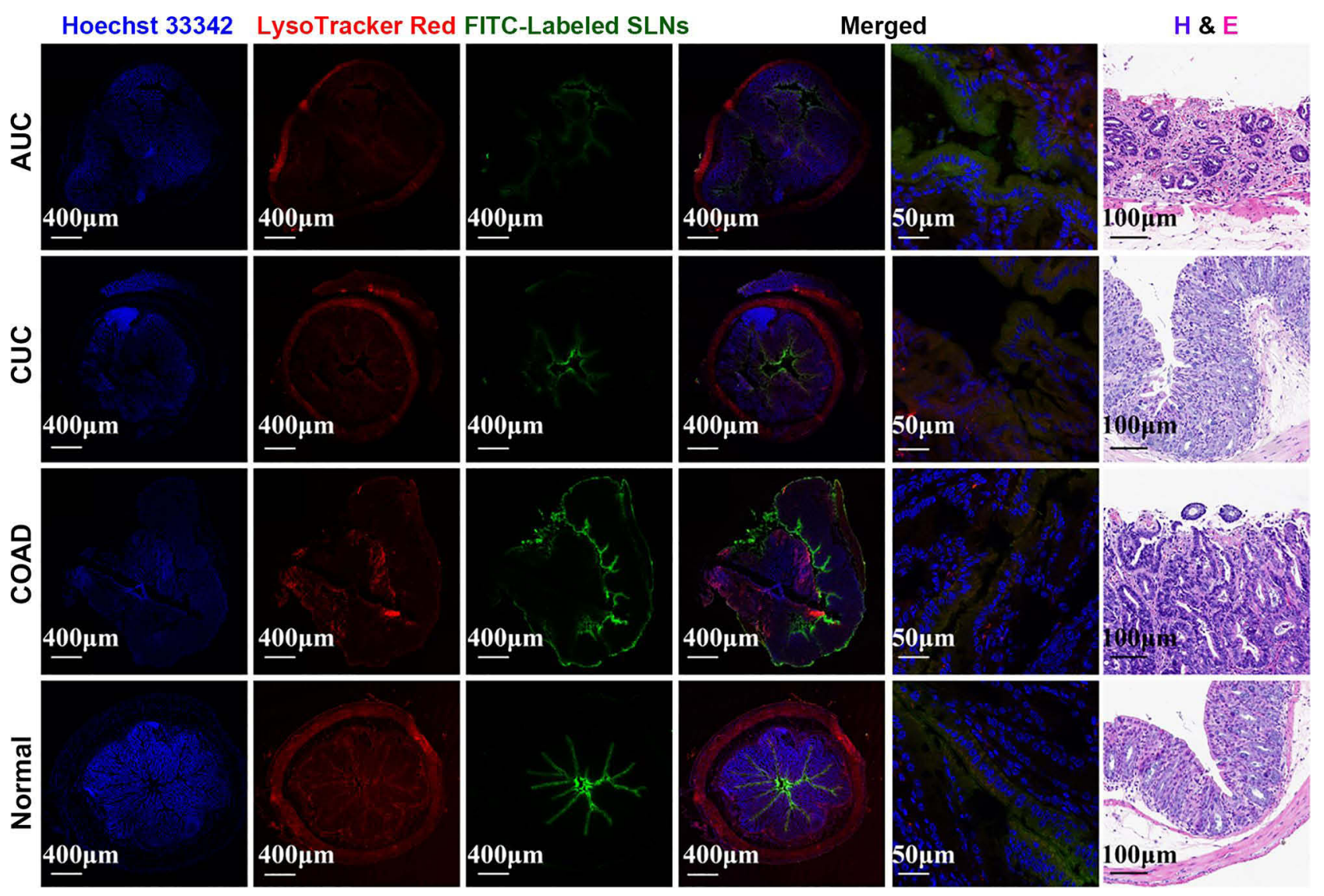

Figure 7 Histologic analysis after MR imaging.

Notes: Confocal laser scanning microscopy images and hematoxylin and eosin-stained images of intestinal issues in different mouse models after enema of FeDM-FITCSLNs. Yellow is colocalization of SLNs and intestinal issues.

Abbreviations: H\&E, hematoxylin-eosin staining; FITC, fluorescein isothiocyanate; AUC, acute ulcerative colitis; CUC, chronic ulcerative colitis; COAD, colon adenocarcinoma.

studies, ${ }^{37}$ SLNs could be preferentially taken up via caveolae to enter the lysosomes. However, few studies have reported SLN transport mechanisms in intestinal lesions.

Boyle et al have proved that inflammatory factors can increase intestinal permeability. For example, interferon gamma (IFN- $\gamma$ ) and TNF- $\alpha$ contribute to intestinal hyperpermeability. ${ }^{38}$ Thus, we speculate that the existence of epithelial defects promotes the adsorption of SLNs; these inflammatory factors integrate with each other to promote SLN absorption in various inflammatory disorders, such as UC. The pathological results and histological findings include acute and chronic inflammatory infiltrates with varying degrees of fibrosis. The CUC group had a disordered arrangement of intestinal glands accompanied by extensive fibrous proliferation. This hinders the absorption of SLNs and makes the duration of enhancement in CUC much shorter than in AUC. Studies have suggested that tumor-infiltrating immune cells disrupt intercellular junctions, which promote $\underline{\mathrm{SLN}}$ absorption in COAD. ${ }^{39}$ In addition, SLNs may also be quickly absorbed to enrich the tumor due to high metabolic activity of malignant tumor cells.

The toxicology of nanoparticles is important, ${ }^{40}$ and data suggest that SLNs are well tolerated. ${ }^{41}$ FeDM-FITC-SLN is a novel class of non-GBCA with an MRI contrast comparable to commercial GBCA. If future studies can provide proof of safety, then FeDM-FITC-SLNs can solve one of the greatest concerns of GBCA: possible long-term toxicity.

The major strengths of this study include its assessment of the differential diagnostic value of intestinal lesions and the standardized disease models. This is the first absorption-based study to investigate differential diagnosis of intestinal lesions by MRI. Moreover, we found that FeDM-FITC-SLN is a promising gadolinium (III)-free alternative. Nonetheless, our study does have some limitations. First, we do not have data on the dose-intensity or optimal doses of FeDM-FITC-SLNs. Second, adenoma and Crohn's disease were not analyzed due to the lack of a suitable model 
system that mimics human disease. Third, the human body is more complicated than an animal model. Thus, in-depth clinical studies are needed.

\section{Conclusion}

In this study, we developed a non-GBCA absorbable by the intestinal wall for the MRI of intestinal lesions. In terms of intestinal bowel diseases, it is obvious that the different absorption levels of FeDM-FITC-SLNs will help in the differential diagnosis and subsequent choice of appropriate treatment. These findings have potential value in studying the impact of FeDM-FITC-SLNs on MR colonography.

\section{Acknowledgments}

This study was supported by Key Research and Development Program of Zhejiang Province (2019C03014), the National Natural Science Foundation of China (81871403), and Zhejiang Provincial Natural Science Foundation of China (LY21H160041).

\section{Disclosure}

Dr Siqi Wang reports a patent preparation method and application of a Fe-levodopa or its ester complex with MRI contrast effect pending to Jihong Sun, Siqi Wang, Jun Ling. The authors report no other conflicts of interest in this work.

\section{References}

1. Lee BC, Lee JY, Kim J, et al. Graphene quantum dots as anti-inflammatory therapy for colitis. Sci Adv. 2020;6(18):eaaz2630. doi:10.1126/sciadv. aaz2630

2. Han C, Xing G, Zhang M, et al. Wogonoside inhibits cell growth and induces mitochondrial-mediated autophagy-related apoptosis in human colon cancer cells through the PI3K/AKT/mTOR/p70S6K signaling pathway. Oncol Lett. 2018;15(4):4463-4470. doi:10.3892/ol.2018.7852

3. Bray F, Ferlay J, Soerjomataram I, et al. Global cancer statistics 2018: GLOBOCAN estimates of incidence and mortality worldwide for 36 cancers in 185 countries. CA Cancer J Clin. 2018;68(6):394-424. doi:10.3322/caac.21492

4. Liu LQ, Li HS, Nie SP, et al. Tea polysaccharide prevents colitis-associated carcinogenesis in mice by inhibiting the proliferation and invasion of tumor cells. Int J Mol Sci. 2018;19(2):506-520. doi:10.3390/ijms19020506

5. Nakase H, Sakuma S, Fukuchi T, et al. Evaluation of a novel fluorescent nanobeacon for targeted imaging of Thomsen-Friedenreich associated colorectal cancer. Int J NanoMedicine. 2017;12:1747-1755. doi:10.2147/IJN.S124174

6. Sethi MK, Fanayan S. Mass spectrometry-based N-glycomics of colorectal cancer. Int J Mol Sci. 2015;16(12):29278-29304. doi:10.3390/ ijms 161226165

7. Thornton E, Morrin MM, Yee J. Current status of MR colonography. Radiographics. 2010;30(1):201-218. doi:10.1148/rg.301095519

8. Luboldt W, Bauerfeind P, Wildermuth S, et al. Colonic masses: detection with MR colonography. Radiology. 2000;216(2):383-388. doi:10.1148/ Radiology.216.2.r00au11383

9. Ajaj W, Pelster G, Treichel U, et al. Dark lumen magnetic resonance colonography: comparison with conventional colonoscopy for the detection of colorectal pathology. Gut. 2003;52(12):1738-1743. doi:10.1136/gut.52.12.1738

10. Kok VC, Yu CC. cancer-derived exosomes: their role in cancer biology and biomarker development. Int J Nanomedicine. 2020;15:8019-8036. doi:10.2147/IJN.S272378

11. Grobner T. Gadolinium-a specific trigger for the development of nephrogenic fibrosing dermopathy and nephrogenic systemic fibrosis? Nephrol Dial Transplant. 2006;21(4):1104-1108. doi:10.1093/ndt/gfk062

12. Marckmann P, Skov L, Rossen K, et al. Nephrogenic systemic fibrosis: suspected causative role of gadodiamide used for contrast-enhanced magnetic resonance imaging. J Am Soc Nephrol. 2006;17(9):2359-2362. doi:10.1681/ASN.2006060601

13. Mathieu P, Coppel Y, Respaud M, et al. Silica coated iron/iron oxide nanoparticles as a nano-platform for T2 weighted magnetic resonance imaging. Molecules. 2019;24(24):4629. doi:10.3390/molecules24244629

14. Ansari MA, Akhtar S, Rauf MA, et al. Sol-of Dy-Substituted Ni0.4Cu0.2Zn0.4 (Fe2-xDyx)O4 nano spinel ferrites and evaluation of their antibacterial, antifungal, antibiofilm and anticancer potentialities for biomedical application. Int J Nanomedicine. 2021;16:5633-5650. doi:10.2147/IJN.S316471

15. Listyarini RV, Gesto DS, Paiva P, et al. Benchmark of density functionals for the calculation of the redox potential of $\mathrm{Fe} 3+/ \mathrm{Fe} 2+$ within protein coordination shells. Front Chem. 2019;7:391-402. doi:10.3389/fchem.2019.00391

16. Miao Y, Xie F, Cen J, et al. Fe3+ @polyDOPA- b -polysarcosine, a T1-weighted MRI contrast agent via controlled NTA polymerization. ACS Macro Lett. 2018;7(6):693-698. doi:10.1021/acsmacrolett.8b00287

17. Yang X, Ren T, Wu N, et al. Controlled release levodopa methylester/benserazide loaded PLGA microspheres ameliorated levodopa-induced dyskinesia by inhibiting Tau/FosB signal pathway in dyskinetic rats. Chin J Neuroimmunol Neurol. 2018;15(4):4463-4470.

18. El-Leithy E, Shaker D, Ghorab M, et al. Evaluation of mucoadhesive hydrogels loaded with diclofenac sodium-chitosan microspheres for rectal administration. AAPS Pharm Sci Tech. 2010;11(4):1695-1702. doi:10.1208/s12249-010-9544-3

19. Ensign L, Cone R, Hanes J. Oral drug delivery with polymeric nanoparticles: the gastrointestinal mucus barriers. Adv Drug Delivery Rev. 2012;64 (6):555-570. doi:10.1016/j.addr.2011.12.009 
20. Mehnert W, Mder K. Solid lipid nanoparticles: production, characterization and applications. Adv Drug Deliv Rev. 2012;64(2-3):83-101. doi:10.1016/j.addr.2012.09.021

21. Wang HY, Ding WX, Peng LJ, et al. Gadolinium-loaded solid lipid nanoparticles for colorectal tumor in MR colonography. JBN. 2020;16 (5):594-602.

22. Dong X, Luo J, Lan P, et al. Magnetic resonance colonography with intestine-absorbable nanoparticle contrast agents in evaluation of colorectal inflammation. Eur Radiol. 2021;31:4615-4624. doi:10.1007/s00330-020-07609-8

23. Sun J, Zhang S, Jiang S, et al. Gadolinium-loaded solid lipid nanoparticles as a tumor-absorbable contrast agent for early diagnosis of colorectal tumors using magnetic resonance colonography. J Biomed Nanotechnol. 2016;12(9):1709-1723. doi:10.1166/jbn.2016.2285

24. Willemsen AE, Lubberman FJ, Tol J, et al. Effect of food and acid-reducing agents on the absorption of oral targeted therapies in solid tumors. Drug Discov Today. 2016;21(6):962-976. doi:10.1016/j.drudis.2016.03.002

25. Sun J, Zheng W, Zhang H, et al. Development of nanoparticle-based magnetic resonance colonography. Magn Reson Med. 2011;65(3):673-679. doi: $10.1002 / \mathrm{mrm} .22654$

26. Yuan H, Miao J, Du YZ, et al. Cellular uptake of solid lipid nanoparticles and cytotoxicity of encapsulated paclitaxel in A549 cancer cells. Int J Pharm. 2008;348:137-145. doi:10.1016/j.ijpharm.2007.07.012

27. Yuan H, Huang LF, Du YZ, et al. Solid lipid nanoparticles prepared by solvent diffusion method in a nanoreactor system. Colloids Surf B Biointerfaces. 2008;61:132-137. doi:10.1016/j.colsurfb.2007.07.015

28. Encinas C, Miltsov S, Otazo E, et al. Synthesis and spectroscopic characterisation of heptamethincyanine NIR dyes for their use in optochemical sensors. Dyes Pigments. 2006;71(1):28-36. doi:10.1016/j.dyepig.2005.05.013

29. Melgar S, Karlsson A, Michaelsson E. Acute colitis induced by dextran sulfate sodium progresses to chronicity in C57BL/6 but not in BALB/c mice: correlation between symptoms and inflammation. Am J Physiol Gastrointest Liver Physiol. 2005;288:1328-1338. doi:10.1152/ ajpgi.00467.2004

30. Okayasu I, Hatakeyama S, Yamada M, et al. A novel method in the induction of reliable experimental acute and chronic ulcerative colitis in mice. Gastroenterology. 1990;98(3):694-702. doi:10.1016/0016-5085(90)90290-H

31. Yu C, Wen XD, Zhang Z, et al. American ginseng attenuates azoxymethane/dextran sodium sulfate-induced colon carcinogenesis in mice. J Ginseng Res. 2015;39(1):14-21. doi:10.1016/j.jgr.2014.07.001

32. Balzer T. Contrast agents for magnetic resonance imaging. 2006.

33. Boehm-Sturm P, Haeckel A, Hauptmann R, et al. Low-molecular-weight iron chelates may be an alternative to gadolinium-based contrast agents for T1-weighted contrast-enhanced MR imaging. Radiology. 2017;286(2):537-546. doi:10.1148/radiol.2017170116

34. Alhamami M, Cheng W, Lyu Y, et al. Manganese-porphyrin-enhanced MRI for the detection of cancer cells: a quantitative in vitro investigation with multiple clinical subtypes of breast cancer. PLoS One. 2018;13(5):e0196998. doi:10.1371/journal.pone.0196998

35. Chai $\mathrm{G}, \mathrm{Xu}$ Y, Chen S,et al. Transport mechanisms of solid lipid nanoparticles across caco-2 cell monolayers and their related cytotoxicology [J]. Acs Appl Mater Interfaces, 2016, 8(9):5929-5940. doi:10.1021/acsami.6b00821. Epub 2016 Feb 23.

36. He B, Shi Y, Liang Y, et al. Single-walled carbon-nanohorns improve biocompatibility over nanotubes by triggering less protein-initiated pyroptosis and apoptosis in macrophages. Nat Commun. 2018;9(1):2393-2414. doi:10.1038/s41467-018-04700-z

37. Wiemann M, Vennemann A, Stintz M, et al. Effects of ultrasonic dispersion energy on the preparation of amorphous SiO2 nanomaterials for in vitro toxicity testing. Nanomaterials. 2018;9(1):11-43. doi:10.3390/nano9010011

38. Boyle KE, Magill-Collins MJ, Newsom SA, et al. Maternal fat-1 transgene protects offspring from excess weight gain, oxidative stress, and reduced fatty acid oxidation in response to high-fat diet. Nutrients. 2020;12(3):767-779. doi:10.3390/nu12030767

39. Kadota K, Yeh YC, Villena-Vargas J, et al. Tumor budding correlates with the protumor immune microenvironment and is an independent prognostic factor for recurrence of stage I lung adenocarcinoma. Chest. 2015;148(3):711-721. doi:10.1378/chest.14-3005

40. Lewinski N, Colvin V, Drezek R. Cytotoxicity of nanoparticles. Small. 2008;4(1):26-49. doi:10.1002/smll.200700595

41. Lim S, Banerjee A, Onyuksel H. Improvement of drug safety by the use of lipid-based nanocarriers. J Controlled Release. 2012;163(1):34-45. doi:10.1016/j.jconrel.2012.06.002

International Journal of Nanomedicine

Dovepress

\section{Publish your work in this journal}

The International Journal of Nanomedicine is an international, peer-reviewed journal focusing on the application of nanotechnology in diagnostics, therapeutics, and drug delivery systems throughout the biomedical field. This journal is indexed on PubMed Central, MedLine, CAS, SciSearch ${ }^{\circledR}$, Current Contents ${ }^{\circledR} /$ Clinical Medicine, Journal Citation Reports/Science Edition, EMBase, Scopus and the Elsevier Bibliographic databases. The manuscript management system is completely online and includes a very quick and fair peer-review system, which is all easy to use. Visit http:// www.dovepress.com/testimonials.php to read real quotes from published authors.

Submit your manuscript here: https://www.dovepress.com/international-journal-of-nanomedicine-journal 